\title{
A Simulation of One Dimensional Contaminant Transport
}

\author{
Roberto Cianci \\ DIME, Università di Genova \\ Piazzale J.F. Kennedy, pad D, \\ Genova, Italy \\ roberto.cianci@unige.it
}

\author{
Agostino Bruzzone \\ DIME, Università di Genova \\ Via all'Opera Pia 15, Genova, \\ Italy \\ ago@itim.unige.it
}

\author{
Roberta Sburlati \\ DICCA, Università di Genova \\ Via Montallegro 1, Genova, \\ Italy \\ roberta.sburlati@unige.it
}

\begin{abstract}
In this note we present some simulations and some analytical solutions, in closed form, of the advectiondispersion equation in one-dimensional domain. These solutions are obtained for not-conservative solutes by considering time-dependent, third type (Robin) boundary condition for first order reaction and linear equilibrium sorption. The Robin boundary condition models a combined production-decay function. The model is useful to describe sources as the contaminant release due to the failure of an underground pipelines or radioactive decay series. The developed analytical model gives rise to analytical solutions not present in the literature. Further, we remark that, for particular values of the rate constants involved in the model, our results furnish values which are in agreement with results present in the literature.
\end{abstract}

\section{Author Keywords}

Simulation, diffusion, Partial differential equation, pollution phenomena, boundary conditions.

\section{ACM Classification Keywords}

I.6.1 Simulation and Modeling (Applications) .1.8 Partial

Differential Equations (Parabolic equations)

\section{INTRODUCTION}

Exact analytical solutions of the advection-dispersion equation are rarely possible if some complex chemical processes such as multi-component reaction chains and nonlinear sorption are considered together. Two- or threedimensional analytical solutions have been provided only for simple first order degradation or decay, zero order production and linear equilibrium sorption, described by a retardation factor. Some particular solutions taking into account nonlinear adsorption/desorption have been developed by using the Green Function Method (GFM) for various plane and linear sources $[1,2,3,4,5,6]$.

Some one-dimensional analytical solutions in closed form have been proposed in the last decades, and attention of scientist in the last few years has been devoted to solutions having time dependent sources. In [7] the authors proposed a library of one-dimensional analytical models that contains

SummerSim-SCSC, 2016 July 24-27, Montreal, Quebec, Canada (c) 2016 Society for Modeling \& Simulation International (SCS) some solutions by considering source decay, and proposed an approach based on Duhamel theorem to construct onedimensional solutions with time dependent boundary conditions and presented some closed form solutions for exponential source decay.

In this contribution, we present a solution method which, under a wide choice of initial and boundary conditions of the simulation model, allows us to get a closed form solution for the concentration by using a superposition method.

The technique of solution here presented are original and they can be useful to match many physical and applicative cases. The general method proposed reduces all the computation to the knowledge to a function representing initial data condition. Further, by using a suitable expression dependent on four arbitrary parameters, we produce a technique that can be easily used to match specific applicative cases, some of which will be presented with some plots.

Moreover, a one-dimensional solution can be used as it is, to model contaminant transport in laboratory columns, pollution in aquifers in which the contamination source extends throughout the saturated zone in both the transverse $\mathrm{y}$ and $\mathrm{z}$ directions, situations where dispersion in $\mathrm{y}$ and $\mathrm{z}$ directions can be ignored and in assessing vertical flow of contaminants in saturated soil $[8,9,10,11,12,13]$.

We use Laplace transform techniques and integral transforms that require the calculation of some non trivial integrals of special functions. The calculations have been performed and closed form solutions are obtained.

\section{THE MODEL}

We write the one-dimensional advection-dispersion equation describing contaminant transport subject to first order decay and linear equilibrium sorption in a semiinfinite as:

$R \frac{\partial C(x, t)}{\partial t}=D \frac{\partial^{2} C(x, t)}{\partial x^{2}}-v \frac{\partial C(x, t)}{\partial x}-\lambda C(x, t)+\gamma$

Here $C(x, t)$ is the contaminant concentration, $D$ is the dispersion coefficient, $v$ is the average space velocity, $\lambda$ is the first order decay constant, $R$ is the retardation factor. We 
assume a saturated homogeneous porous medium with constant and uniform fluid flow and dispersion coefficients. Further $\gamma$ represents the rate constant for zero-order production in the domain.

The following initial and boundary conditions are assumed:

$$
\begin{aligned}
& C(x, 0)=0, \\
& -D \frac{\partial C(0, t)}{\partial x}+v C(0, t)=v g(t), \quad \frac{\partial C(\infty, t)}{\partial x}=0
\end{aligned}
$$

where $g(t)$ represents a general time-dependent function.

We give now some details of the mathematical procedure. Firstly, we introduce the new variable $c(x, t)$ :

$$
C(x, t)=\exp \left(\frac{v x}{D}\right) \exp (-\lambda t) c(x, t)
$$

So doing, the boundary conditions will change and the problem which is of the third type (Robin) for $C(x, t)$ becomes of the second type for $c(x, t)$. Actually, the main equation becomes:

$$
\frac{\partial c(x, t)}{\partial t}=D \frac{\partial^{2} c(x, t)}{\partial x^{2}}-v \frac{\partial c(x, t)}{\partial x}
$$

Concerning boundary conditions, by deriving this expression with respect to $x$, we get:

$$
\begin{aligned}
\frac{\partial C(x, t)}{\partial x}= & \frac{v \exp \left(\frac{v x}{D}\right) \exp (-\lambda t) c(x, t)}{D}+ \\
& +\exp \left(\frac{v x}{D}\right) \exp (-\lambda t)\left(\frac{\partial c(x, t)}{\partial x}\right)
\end{aligned}
$$

By substituting, this equation can be rewritten as:

$$
\frac{\partial C(x, t)}{\partial x}=\frac{v C(x, t)}{D}+\exp \left(\frac{v x}{D}\right) \exp (-\lambda t)\left(\frac{\partial c(x, t)}{\partial x}\right)
$$

And, by evaluating this relation in $x=0$, we have:

$$
\frac{\partial C(0, t)}{\partial x}-\frac{v C(0, t)}{D}=\exp (-\lambda t)\left(\frac{\partial c(0, t)}{\partial x}\right)
$$

By recalling that:
$-D \frac{\partial C(0, t)}{\partial x}+v C(0, t)=v g(t)$

we get:

$\frac{\partial C(0, t)}{\partial x}-\frac{v C(0, t)}{D}=-\frac{v g(t)}{D}$

Now we set $\frac{v g(t)}{D}=f(t)$, and so we obtain:

$\frac{\partial C(0, t)}{\partial x}-\frac{v C(0, t)}{D}=-f(t)$

together with the expression:

$\left(\frac{\partial c(0, t)}{\partial x}\right)=-f(t) \exp (\lambda t)$

So the final general problem, with the new boundary conditions, is a one-dimensional problem subject to secondtype boundary conditions.

Solving with Laplace transform in time, the general solution is the following:

$$
c(x, t)=-\frac{v n_{1}(x, t)}{2}+\frac{\sqrt{D} \exp \left(-\frac{v x}{2 D}\right) \exp \left(\frac{-v^{2} t}{4 D}\right) n_{2}(x, t)}{\sqrt{\pi}}
$$

where we have introduced the two functions:

$$
n_{1}(x, t)=\int_{0}^{t} f(q) \exp (\lambda q) \operatorname{erfc}\left(\frac{v \sqrt{t-q}+\frac{x}{\sqrt{t-q}}}{2 \sqrt{D}}\right) d q
$$

and

$n_{2}(x, t)=\int_{0}^{t} \frac{f(q) \exp (\lambda q) \exp \left(\frac{q v^{2}}{4 D}\right) \exp \left(-\frac{x^{2}}{4 D(t-q)}\right)}{\sqrt{t-q}} d q$

The complementary error function $\operatorname{erfc}(x)$ is defined as

$$
\operatorname{erfc}(x)=1-\operatorname{erf}(x) d t
$$

where the error function $\operatorname{erf}(x)$ is 
$\operatorname{erf}(x)=\frac{2}{\sqrt{\pi}} \int_{0}^{t} \exp \left(-t^{2}\right) d t$

With these expression for the functions $n_{1}(x, t), n_{2}(x, t)$, it is possible to check that these two integrals are related by the following equality:

$$
\frac{\partial n_{1}(x, t)}{\partial x}=-\frac{\exp \left(-\frac{t v^{2}}{4 D}\right) \exp \left(-\frac{v x}{2 D}\right) n_{2}(x, t)}{\sqrt{\pi D}}
$$

The integral solution becomes:

$$
c(x, t)=-\frac{v n_{1}(x, t)}{2}-D \frac{\partial n_{1}(x, t)}{\partial x}
$$

And, finally, by substituting, we get

$$
C(x, t)=\exp \left(\frac{v x}{D}\right) \exp (-\lambda t)\left(-\frac{v n_{1}(x, t)}{2}-D \frac{\partial n_{1}(x, t)}{\partial x}\right)
$$

We remark that the explicit solution is obtained only in terms of the $n_{1}(x, t)$ integral.

In order to carry on our discussion, we have to specify the function $g(t)$ for our study.

\section{THE PROPOSED CASE}

We consider the case

$$
g(t)=C_{0} y\left(1-e^{-\lambda_{p} t}\right)+C_{0} e^{-\lambda_{s} t}
$$

which is able to simulate the time evolution of a pollution event since the parameters $y, \lambda_{p}, \lambda_{s}$ can be freely chosen.

With this assumption, we are able to compute the integrals, so obtaining some new analytical solutions proposed in a semi-finite domain. For the development of the simulation techniques we used Maple symbolic calculation software and MaplaSim package.

The expressions furnishing the values of the functions $n_{1}(x, t), n_{2}(x, t)$ become:

$$
\begin{aligned}
n_{1}(x, t) & =\int_{0}^{t} \frac{v}{D} C_{0} y\left(1-e^{-\lambda_{p} q}\right) d q+ \\
& +\int_{0}^{t} C_{0} e^{-\lambda_{s} q} \exp (\lambda q) \operatorname{erfc}\left(\frac{v \sqrt{t-q}+\frac{x}{\sqrt{t-q}}}{2 \sqrt{D}}\right) d q
\end{aligned}
$$

$$
\begin{aligned}
& n_{2}(x, t)=\frac{v}{D} C_{0} y \int_{0}^{t} \frac{\left(1-e^{-\lambda_{p} q}\right)}{\sqrt{t-q}} d q+ \\
& +C_{0} \int_{0}^{t} \frac{e^{-\lambda_{s} q} \exp (\lambda q) \exp \left(\frac{q v^{2}}{4 D}\right) \exp \left(-\frac{x^{2}}{4 D(t-q)}\right)}{\sqrt{t-q}} d q
\end{aligned}
$$

These integral can be computed by using special functions techniques.

The final solution is:

$$
C(x, t)=C_{0} y(A(x, t)+B(x, t))+C_{0} E(x, t)
$$

where we have set:

$$
\begin{aligned}
A(x, t)= & \frac{v}{v+u} \exp \left(\frac{(v-u) x}{2 D_{x}}\right) \operatorname{erfc}\left(\frac{x-t u}{2 \sqrt{D_{x} t}}\right)+ \\
& \frac{v}{v-u} \exp \left(\frac{(v+u) x}{2 D_{x}}\right) \operatorname{erfc}\left(\frac{x+t u}{2 \sqrt{D_{x} t}}\right)+ \\
& +\frac{v^{2}}{2 \lambda D_{x}} \exp \left(\frac{v x}{D_{x}}\right) \exp (-\lambda t) \operatorname{erfc}\left(\frac{x+t v}{2 \sqrt{D_{x} t}}\right)
\end{aligned}
$$

$$
\begin{aligned}
B(x, t) & =-\exp \left(-\lambda_{p} t\right)\left(\frac{v}{v+w} \exp \left(\frac{(v-w) x}{2 D_{x}}\right) \operatorname{erfc}\left(\frac{x-t w}{2 \sqrt{D_{x} t}}\right)\right) \\
& -\exp \left(-\lambda_{p} t\right)\left(\frac{v}{v-w} \exp \left(\frac{(v+w) x}{2 D_{x}}\right) \operatorname{erfc}\left(\frac{x+t w}{2 \sqrt{D_{x} t}}\right)\right) \\
& -\frac{v^{2}}{2(\lambda-B) D_{x}} \exp \left(\frac{v x}{D_{x}}\right) \exp (-\lambda t) \operatorname{erfc}\left(\frac{x+t v}{2 \sqrt{D_{x} t}}\right)
\end{aligned}
$$

$$
\begin{aligned}
E(x, t) & =\exp \left(-\lambda_{s} t\right)\left(\frac{v}{v+W} \exp \left(\frac{(v-W) x}{2 D_{x}}\right) \operatorname{erfc}\left(\frac{x-t W}{2 \sqrt{D_{x} t}}\right)\right)+ \\
& +\exp \left(-\lambda_{s} t\right)\left(\frac{v}{v-W} \exp \left(\frac{(v+W) x}{2 D_{x}}\right) \operatorname{erfc}\left(\frac{x+t W}{2 \sqrt{D_{x} t}}\right)\right) \\
& +\frac{v^{2}}{2(\lambda-S) D_{x}} \exp \left(\frac{v x}{D_{x}}\right) \exp (-\lambda t) \operatorname{erfc}\left(\frac{x+t v}{2 \sqrt{D_{x} t}}\right)
\end{aligned}
$$


The quantities $u, w$ and $W$ are defined as follows:

$u=\sqrt{4 D_{x} \lambda+v^{2}}, w=\sqrt{4 D_{x}\left(\lambda-\lambda_{p}\right)+v^{2}}$

$W=\sqrt{4 D_{x}\left(\lambda-\lambda_{s}\right)+v^{2}}$

First of all, we remark that, when $\lambda_{\mathrm{p}}=0$ and $\lambda_{\mathrm{s}}=0$, or when $\lambda_{\mathrm{p}} \rightarrow \infty$, this solution gives rise to solutions already present in

[7]. Now we study and plot some specific cases.

THE NUMERICAL RESULTS

We now study the new solution for various choices of the parameters, namely:

1. Let $g(t)=C_{0} y\left(1-e^{-\lambda_{p} t}\right)+C_{0} e^{-\lambda_{s} t}$

for $C_{0}=850, C_{0}(\infty)=350, \lambda_{p}=0,00221 / \mathrm{sec}$,

in the cases:

$\lambda_{s}=0.00015 / \mathrm{sec}, \lambda_{s}=0.00008 / \mathrm{sec}$

$\lambda_{s}=0.000012 / \mathrm{sec}$

2. Let $g(t)=C_{0} e^{-\lambda_{s} t}$ for

$C_{0}=850, C_{0}(\infty)=350, \lambda_{p}=0$,

in the cases:

$\lambda_{s}=0.00008 / \mathrm{sec}, \lambda_{s}=0.0012 / \mathrm{sec}$

$\lambda_{s}=0.0022 / \mathrm{sec}$

3. Let $g(t)=C_{0} y\left(1-e^{-\lambda_{p} t}\right)+C_{0}$

for $C_{0}=850, C_{0}(\infty)=350, \lambda_{s}=0$,

in the cases:

$\lambda_{p}=0.0008 / \mathrm{sec}, \lambda_{s}=0.0012 / \mathrm{sec}$

$\lambda_{s}=0.0022 / \mathrm{sec}$

In the figures here presented there are the plots of the concentration $C(x, t)$ for these three cases.

Figure. 1.

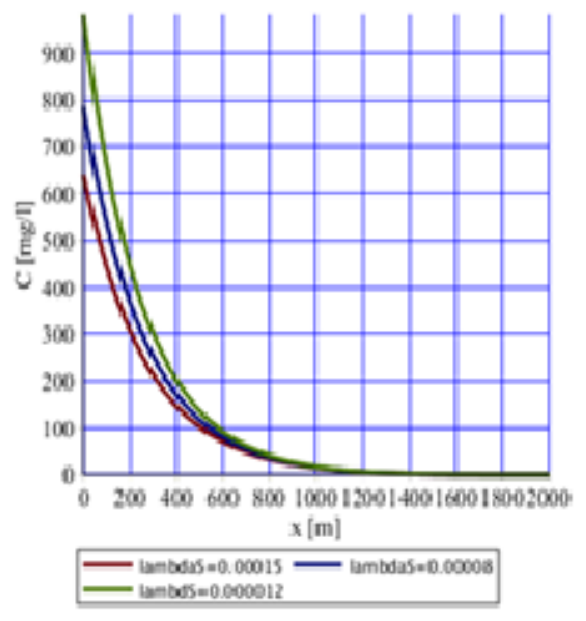

Figure 2.

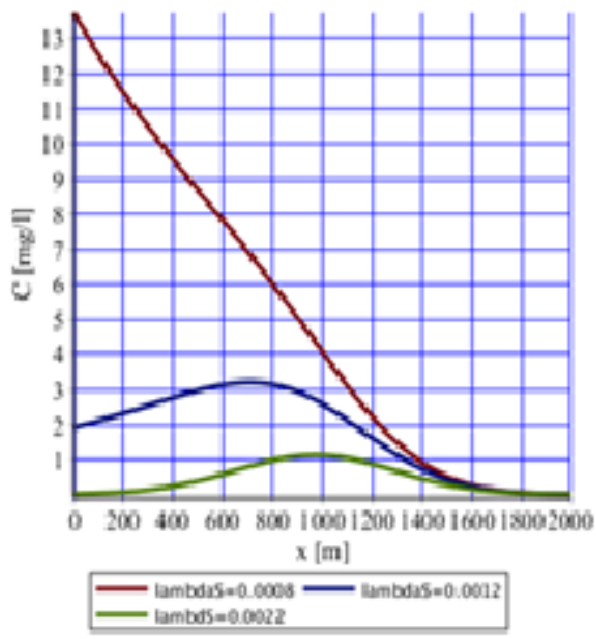


Figure 3.

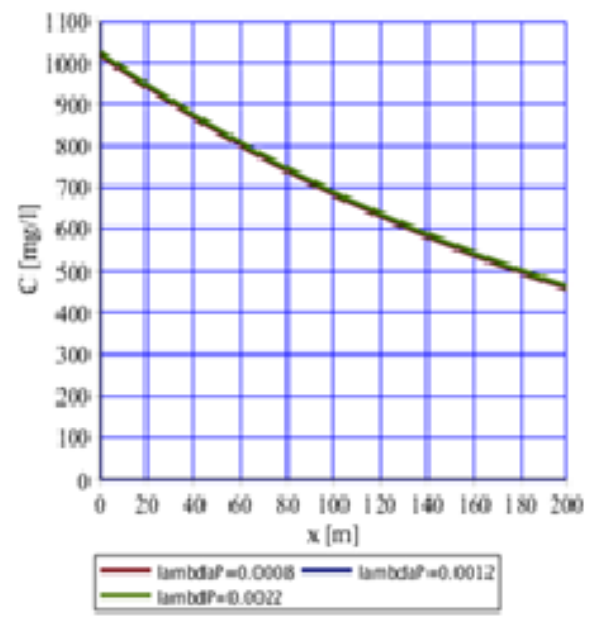

We observe that the new model can describe source as a combined production-consumption and time dependent function where source production and decay are not correlated to each other and, by properly setting the parameters, it is possible to simulate two solutions [7] given by two different problems.

\section{CONCLUSION}

The contaminant transport in porous media has a crucial role in environmental engineering science and petroleum engineering.

Even if Advection-Dispersion Equations very well describe the problem, it is not easy to derive analytical solutions if chemical processes take place too.
On the other hand, analytical solutions in close or open form are provided from the literature for simpler cases.

The one dimensional model here proposed can be useful to describe tank or pipeline failure since it describe the source as a combined production-consumption and time dependent function given as a third type boundary condition.

Finally, the new model can describe source as a combined production-consumption and time dependent function where source production and decay are not correlated to each other and, by properly setting the parameters, it is possible to simulate solution present in the literature.

\section{REFERENCES}

1. Sagar, B., 1982.. ASCE J. Hydraul. Div., 108(HY1), 47-62.

2. Yeh, G.T., AT123,1981.. Oak Ridge National Laboratory, Report. ORNL-5602, Oak Ridge, Tennessee, USA. 83 pp

3. Wexler, E.J.,1992., Techniques of water-Resources Investigations, Book 3, Chap. B7.

4. Batu, V., 1993.. Water Resour. Res. 29, 2881-2892.

5. Batu, V., 1996.. J. Hydrol. 174, 57-82.

6. Neville, C. J., 1994., S.S. Bethesda, MD, USA: Papadopus \& Associates.

7. Van Genuchten, M. T.H., Alves, W.J., 1982.. U.S. Department of Agricolture Technical Bullettin No. 1661, USDA, Riverside, Calif.

8. Cianci R., Bruzzone A., Sburlati R., Acta Appl Math (2014) (132), p. 213-224

9. Leji, F.J., Skaggs T.H., van Genuchten M.T., 1991. Water Resour. Res. 27(10), 2719-2733.

10. Leji, F.J., Toride, N., van Genuchten, M, Th., 1993.. J. Contam. Hydrol. 151, 193228.

11. Ogata, A., Banks, R.B., 1961. U.S. Geol. Surv. Prof. Pap. 411, A1-A7.

12. Park, E., Zhan, H., 2001. J. Contam Hydrol. 53, 41-61.

13. Van der Zee, S.E.A.T.M., 1990.. Water Resour. Res. 26, 273-278. 